\title{
The Effects of Morphine, Baclofen, and Buspirone Alone and in Combination on Schedule-Controlled Responding and Hot Plate Antinociception in Rats ${ }^{[}$
}

\author{
Jenny L. Wilkerson, Jasmine S. Felix, Luis F. Restrepo, Mohd. Imran Ansari, Andrew Coop, \\ and Lance R. McMahon
}

Department of Pharmacodynamics, College of Pharmacy, University of Florida, Gainesville, Florida (J.L.W., J.S.F., L.F.R., L.R.M.) and Department of Pharmaceutical Sciences, School of Pharmacy, University of Maryland, Baltimore, Maryland (M.I.A., A.C.)

Received December 15, 2018; accepted June 14, 2019

\begin{abstract}
Better therapeutic options are needed for pain. Baclofen, buspirone, and morphine are characterized as having analgesic properties. However, little is known about potential interactions between analgesic effects of these drugs when combined. Furthermore, it is not known if the magnitude of these potential interactions will be similar for all drug effects. Thus, we tested the effects of these drugs alone and in combination for their capacity to produce thermal antinociception and to decrease food-maintained responding. Four male and four female Sprague-Dawley rats responded for food under a fixed-ratio 10 schedule; afterward they were immediately placed on a $52^{\circ} \mathrm{C}$ hot plate. Morphine, baclofen, and buspirone were examined alone and in 1:1 combinations, based upon $\mathrm{ED}_{50}$ values. Morphine and baclofen effects were evaluated with the opioid antagonist naltrexone and the $\mathrm{GABA}_{\mathrm{B}}$ antagonist (3-Aminopropyl)(diethoxymethyl)phosphinic
\end{abstract}

\section{Introduction}

Currently the United States is in the midst of an opioid overdose epidemic. It is likely that opioids will continue to be needed for treating pain for the foreseeable future, especially severe acute pain and cancer pain (Fields, 2011). The low dose combination of drugs from different pharmacological classes, including opioids, is a recognized strategy to circumvent potential untoward side effects (Wilkerson et al., 2016, 2017). Although there is considerable interest in the development of novel therapeutics to treat pain, with a keen interest on minimizing drug abuse liability, the clinical fruition of these alternatives takes many years, often with high costs that are passed down to the patient. Hence, there is notable interest in repurposing drugs that are already US Food and Drug Administration (FDA) approved for new and innovative uses (Corsello et al., 2017). Thus, there may be suitable drugs already available that may lessen the burden of opioid use for pain management by producing stable and adequate pain

This work was supported by the National Institutes of Health National Institute on Drug Abuse DA25267.

https://doi.org/10.1124/jpet.118.255844.

S This article has supplemental material available at jpet.aspetjournals.org. acid (CGP35348), respectively. Morphine, baclofen, and buspirone dose dependently decreased operant responding, with the calculated $\mathrm{ED}_{50}$ values being $7.09,3.42$, and $0.57 \mathrm{mg} / \mathrm{kg}$, respectively. The respective antinociception $\mathrm{ED}_{50}$ values were $16.15,8.75$, and $2.20 \mathrm{mg} / \mathrm{kg}$. Analysis of 1:1 combinations showed the effects of morphine plus baclofen to decrease schedule-controlled responding and to produce thermal antinociception were synergistic. Effects of morphine plus buspirone and baclofen plus buspirone to decrease schedule-controlled responding were additive. Effects of the two combinations to produce thermal antinociception were synergistic. Naltrexone and CGP35348 antagonized the effects of morphine and baclofen, respectively. Synergistic antinociceptive effects, in conjunction with additive effects on food-maintained responding, highlight the therapeutic utility of opioid and non-opioid drug combinations.

ABBREVIATIONS: ANOVA, analysis of variance; CL, confidence limits; FDA, Food and Drug Administration; FR, fixed ratio; 5-HT, serotonin; MPE, percent maximum possible effect; Zmix, mixture potency; Zadd, additive potency. 
Previous results suggest that the addition of baclofen to morphine may enhance morphine-induced thermal antinociception in mice and ferrets (Suzuki et al., 2005). Likewise, the combination of buspirone and morphine was found to produce additive thermal antinociceptive effects in non-human primates ( $\mathrm{Li}$ et al., 2011). Additionally, the addition of buspirone to morphine was found to increase morphine-induced thermal antinociception in rats (Haleem and Nawaz, 2017). Here we sought to systematically examine respective pharmacological interactions and to quantify the potency of these drugs alone and in combination, based upon their behavior modifying effects. Furthermore, it is not known if the magnitude of these potential interactions will be the same or different for all drug effects. Either synergistic or additive antinociceptive effects in conjunction with either additive or subadditive behaviorally disruptive effects, respectively, may warrant further development of specific drug combinations as pain therapeutics. Thus, differential behavior modifying effects in this manner may produce an ideal therapeutic window that would allow for the therapeutic use of drug combinations with minimal adverse effects (Foucquier and Guedj, 2015).

One way to examine drugs preclinically for antinociceptive effects is via the hot plate test. The hot plate test specifically examines reflexive pain transmission and is mediated primarily by supraspinal pain processing (Eddy and Leimbach, 1953). Further drug-induced disruptions in behavior can be assessed through measurement of schedule-controlled operant responding for food, which is sensitive to drug-induced disruptions in behavior from numerous drug classes that act in the central nervous system (McMahon and France, 2002; Cunningham and McMahon, 2011). Thus, the present study tested the effects of these drugs alone and in combination in rats for their capacity to produce thermal antinociception and to decrease schedule-controlled responding for food. Another goal of the present study was to examine the extent by which the effects of morphine drug combinations (i.e., morphine plus baclofen, morphine plus buspirone) are mediated by opioid receptors. This was accomplished by combining the opioid receptor antagonist naltrexone with morphine by itself, as well as with the morphine drug combinations. In addition to opioid receptor involvement, we also sought to examine the extent that $\mathrm{GABA}_{\mathrm{B}}$ receptors mediate baclofen drug combination effects (i.e., baclofen plus morphine, baclofen plus buspirone). This was likewise accomplished by combining the $\mathrm{GABA}_{\mathrm{B}}$ receptor antagonist CGP35348 with baclofen by itself, as well as with the baclofen drug combinations.

\section{Materials and Methods}

\section{Animals}

Adult male $(n=4)$ and female $(n=4)$ Sprague-Dawley rats (247-279 g upon arrival; Jackson Laboratory, Charleston, SC) were singly housed in a temperature $\left(20-22^{\circ} \mathrm{C}\right)$-, humidity $(55 \% \pm 10 \%)$, and light-controlled (12-hour light/dark; lights on at 0600) facility that was approved by the Association for Assessment and Accreditation of Laboratory Animal Care. Water was available ad libitum. Rats were food restricted to $90 \%$ of their free-feeding body weight, with access to food (Dustless Precision Pellets Grain-Based Rodent Diet; Bio-Serv, Frenchtown, NJ) 30 minutes following daily experimental sessions, as well as to food during experimental sessions as described below. Animal protocols were approved by the Institutional Animal Care and Use Committee at the University of Florida and were in accordance with the National Institutes of Health Guide for the Care and Use of
Laboratory Animals (National Research Council, 2011). All studies involving animals are reported in accordance with the ARRIVE guidelines for reporting experiments involving animals (Kilkenny et al., 2010; McGrath et al., 2010).

\section{Drugs}

Morphine sulfate pentahydrate (morphine) and naltrexone hydrochloride (naltrexone) were obtained from the National Institute on Drug Abuse Research Technology Branch (Rockville, MD). Baclofen and buspirone were obtained from Sigma-Aldrich (St. Louis, MO). CGP35348 was synthesized as described (Froestl et al., 1995). All drugs were dissolved in sterile saline, and sterile saline served as the vehicle in all experiments. Drugs and vehicle were administered intraperitoneally.

\section{Schedule-Controlled Responding}

Apparatus. Operant conditioning chambers were $25 \mathrm{~cm}$ long, $31 \mathrm{~cm}$ high, and $25 \mathrm{~cm}$ wide, as commercially supplied by Med Associates (Fairfax, VT). Each chamber was equipped with two centrally mounted, 5 -cm-long levers located $9 \mathrm{~cm}$ from the chamber floor and $3 \mathrm{~cm}$ from either wall. When operated, a pellet dispenser delivered a 45-mg food pellet (soy-free PJAI; Test Diet, St. Louis, MO) into a pellet trough, which was centrally mounted between the two levers. Located above each lever was a stimulus light. Houselights were centrally mounted on the ceiling. All chambers were equipped with a fan, which supplied ventilation and white noise.

\section{Operant Procedures}

Rats were trained to lever press 7 days a week, as previously described (McMahon and Cunningham, 2001), with the operant procedure modified for food reinforcement. Initially rats were placed in operant conditioning chambers for 30-90 minutes, under a fixed ratio (FR)1 schedule, where one lever press on the lever designated by illumination of the light above the lever resulted in delivery of one 45-mg pellet; the second lever was inactive. Rats could receive a maximum of 50 pellets; once response rate (responses per second) during each of three consecutive sessions deviated by no more than $\pm 20 \%$ of the 3-day running average, regardless of rate, the FR was increased in the following increments: FR3, FR6, FR10. Responding under the FR10 schedule continued until response rate was stable, defined as three consecutive sessions that deviated by no more than $\pm 20 \%$ of the 3 -day running average. Thereafter, sessions were divided into consecutive, discrete, 20 -minute cycles. Each cycle began with a 15-minute timeout during which the stimulus light was not illuminated and responses on the lever had no programmed consequence. The timeout was followed by a 5 -minute period during which food pellets were available under the FR10 schedule. Each session consisted of up to six cycles. Completion of 10 responses resulted in delivery of a food pellet; a maximum of 10 pellets could be delivered per cycle. Rats received vehicle or drug injections within the first minute of a given cycle. Drug tests were initiated once responding stabilized,, i.e., responding during each of three consecutive sessions deviated by no more than $\pm 20 \%$ of the 3 -day running average calculated for all cycles in all sessions.

\section{Hot Plate Test}

Antinociceptive testing was performed in the hot plate test, as previously described (Ignatowska-Jankowska et al., 2015), but modified for rats. Rats were placed on a heated $\left(52^{\circ} \mathrm{C}\right)$ enclosed Hot Plate Analgesia Meter (Columbus Instruments, Columbus, $\mathrm{OH}$ ), and latency to jump or lick/shake the back paws was determined. If there was no response within 60 seconds, the rat was removed from the apparatus.

\section{Experimental Design}

Immediately following one 20-minute cycle in the operant conditioning procedure, rats were placed on the hot plate and tested for 
their latency to respond to the thermal stimulus. After hot plate testing, rats received injections of either vehicle or a drug dose and returned to the operant conditioning chamber for testing in the next cycle. This repeated testing in both the hot plate and operant conditioning chambers occurred a total of six consecutive 20-minute cycles per drug test session. As shown in Supplemental Fig. 1, exposure to the hot plate did not modify operant response rates, and vice versa, through six consecutive 20 -minute cycles. After each drug test session, rats received a minimum 48-hour washout period before receiving the next drug. During these washout periods, rats were given daily sessions consisting of three to six cycles to lever press for food, without being exposed to the hot plate apparatus.

\section{Drug Combinations}

When the mean effect of a drug to reduce food-maintained responding or to produce antinociception was greater than $50 \%$, the $\mathrm{ED}_{50}$ values and corresponding $95 \%$ confidence limits (CL) were calculated using linear regression, where slopes were allowed to vary, according to Tallarida (2001). Doses for the drug combinations were based upon these calculated $\mathrm{ED}_{50}$ values for drugs to reduce schedulecontrolled responding for food. Specifically, the log base 2 of a drug's $\mathrm{ED}_{50}$, the $\mathrm{ED}_{50}$, as well as the (1/2) $\mathrm{ED}_{50},(1 / 4) \mathrm{ED}_{50}$, and (1/8) $\mathrm{ED}_{50}$ of a drug to decrease schedule-controlled responding were used as experimental doses, which were tested in equipotent combination in both assays.

\section{Data Analysis}

A within-subject design was used to test dose and dose combinations and the order was nonsystematic. Dose-response curves were determined twice for each assay, once at the beginning of experimental testing and again at the end of the experimental testing. The first determination was used for all experimental calculations, and the second determination was used to examine the development of drug tolerance. Hot plate data were converted to percent maximum possible effect (\% MPE) with the following equation: ([(experimental test value - baseline value $) /($ maximum test value - baseline value $)] \times 100$ ) and plotted versus log dose values. Response rates were expressed as a percentage of control for each rat, defined as the mean response rate from the previous three nondrug sessions, with each session defined as the individual cycles for that session averaged together. In each experiment, male and female data were analyzed together in all further data analyses. If the mean effect of a drug did not produce a $50 \%$ or greater effect, an $\mathrm{ED}_{50}$ value was not generated. Potency ratios and $95 \% \mathrm{CL}$ were calculated as the ratio of $\mathrm{ED}_{50}$ values calculated from the dose-response curves, and a potency ratio not including 1 within the 95\% CL indicated a statistically significant difference in potency. In the CGP35348 antagonism study on the effects of the combination of baclofen and buspirone on thermal hotplate latency, $\mathrm{ED}_{50}$ and potency ratio values could not be calculated, thus an unpaired Student's $t$ test was used to compare potency effects. The theoretical additive $\mathrm{ED}_{50}$ value of the combined drugs was calculated from the individual dose-response curves to determine synergistic, additive, or subadditive interactions. The combination was assumed to equal the sum of the effects of each drug. The experimentally derived $\mathrm{ED}_{50}$ values $(\mathrm{Zmix}$ ) from the dose-response curves of the ratios were compared with the predicted additive $\mathrm{ED}_{50}$ values (Zadd). If the empirically derived value and the theoretical value did not differ, the interaction was considered additive (Tallarida, 2001, 2006). The degree of effect (i.e., synergy or additivity) in both assays for a drug combination was calculated with the following equation: [(Hot plate Zadd - Hot plate Zmix)/(Rate Zadd - Rate Zmix)], and modified from Tallarida (2001). All doseresponse data were analyzed using a one-way repeated-measures analysis of variance (ANOVA). A Bonferroni comparison was used for post hoc analysis following a significant ANOVA $(P<0.05)$. Comparisons of initial and rederived dose-response curves were analyzed using a two-way repeated-measures ANOVA. The computer program GraphPad Prism version 6.0 (GraphPad Software Inc., San Diego, CA) was used in all statistical analyses. All data are expressed as the mean \pm S.E.M.

\section{Results}

Effects of Morphine and Baclofen on Schedule-Controlled Responding and Latency to Respond to Thermal Stimuli. Figure 1 shows the effects of morphine and baclofen on rate of FR10 responding for food and latency to respond in the hot plate test. Repeated injections of vehicle did not alter rates of responding for food (Supplemental Fig. 1A; the range of absolute rate of responding was $0.85-0.96$ responses/s) or latency to respond to thermal stimulus (Supplemental Fig. 1B; 13.5-17.7 seconds). Thus, stable baselines were obtained across the six cycles in both schedule-controlled responding $\left(F_{7,35}=1.4 ; P=0.22\right)$ as well as nociceptive latency $\left(F_{7,35}=\right.$ 2.7; $P=0.43$ ). Morphine and baclofen individually dose dependently decreased the rate of food-maintained responding (Fig. $1 \mathrm{~A} ; F_{7,21}=13.7, P<0.001$ ) and (Fig. 1B; $\left.F_{7,28}=27.9, P<0.0001\right)$. The $\mathrm{ED}_{50}$ values of morphine and baclofen to reduce operant responding were 7.09 (95\% CL: $3.60-10.58)$ and 3.42 (95\% CL: $2.07-4.77) \mathrm{mg} / \mathrm{kg}$, respectively. We next assessed the dose-response relationship of equi-effective doses of each drug to reduce schedulecontrolled responding, in combination, and as expected, found a leftward shift in the dose-response relationship compared with either compound administered alone (Fig. 1, A and B). Analysis of the mixture potency (i.e., Zmix) and additive potency (i.e., Zadd) of the combination of morphine and baclofen revealed a synergistic interaction between these drugs. The calculated experimental Zmix [3.73 $(2.86-4.00) \mathrm{mg} / \mathrm{kg}$ ] was significantly less than the calculated theoretical Zadd [5.55 $(5.28-5.82) \mathrm{mg} / \mathrm{kg}]$.

Figure 1 also shows the effects of morphine and baclofen, administered separately and together, on the latency to respond in the hot plate test. Both baclofen and morphine dose dependently increased the latency to respond to thermal stimulus [(Fig. 1C; $F_{7,21}=49.3, P<0.0001$ ), (Fig. $1 \mathrm{D} ; F_{7,35}=$ $36.9, P<0.0001$ ), respectively]. The $\mathrm{ED}_{50}$ values of morphine and baclofen to produce thermal antinociception were 16.15 (95\% CL: 12.69-20.56) and 8.75 (95\% CL: 6.03-12.72) mg/kg, respectively. Operant responding was 2.28 -fold more sensitive to the effects of morphine than was thermal nociception [potency ratio: 2.28 (95\% CL: 1.94-3.52)] and 2.56-fold more sensitive to the effects of baclofen than was thermal nociception [potency ratio: 2.56 (95\% CL: 2.21-2.91)]. We next assessed the dose-response relationship of equi-effective doses of each drug in combination from Fig. 1, A and B, in the hot plate assay and found a leftward shift in the doseresponse relationship compared with either compound administered alone (Fig. 1, C and D). Analysis of the mixture potency and additive potency of the combination of baclofen and morphine revealed a synergistic interaction between these drugs. The calculated experimental Zmix [6.49 $(5.57-7.41) \mathrm{mg} / \mathrm{kg}]$ was significantly less than the calculated theoretical Zadd [13.54 (12.90-14.18) mg/kg)]. The degree of effects from the combination of morphine and baclofen was 3.87 -fold higher in the increase of latency to respond to thermal stimulus than the decrease of foodmaintained responding. 

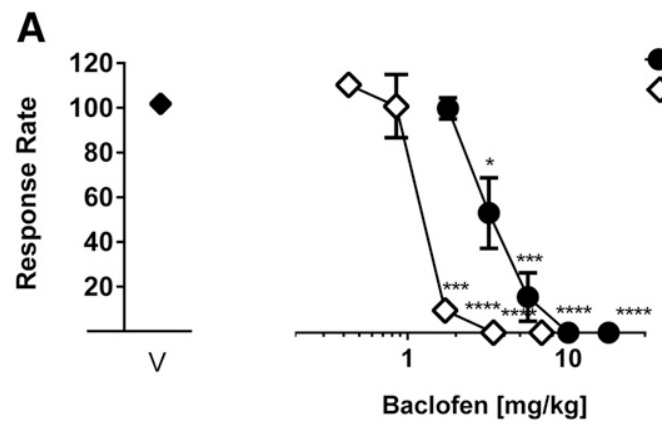

B

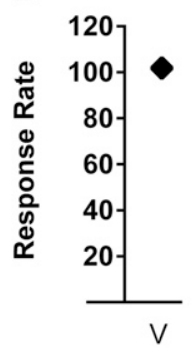

Baclofen

1:1 Baclofen/ Morphine

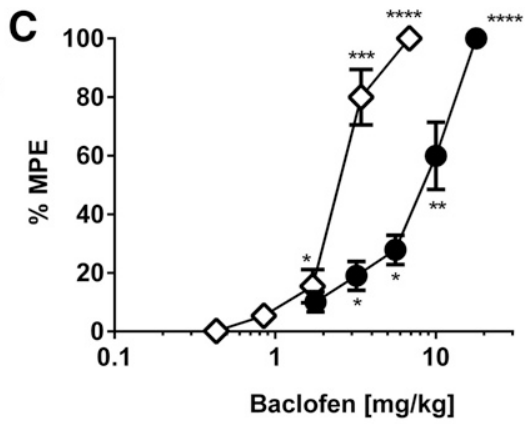

D

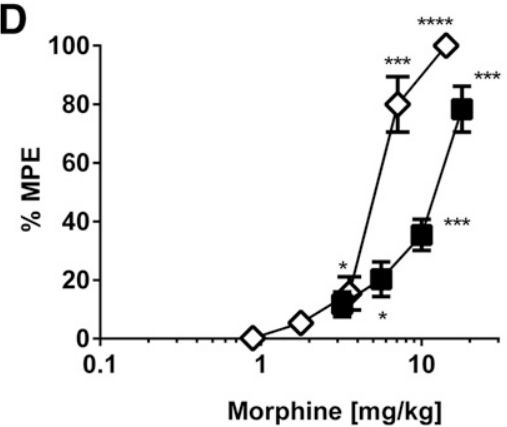

Fig. 1. Systemic baclofen and morphine decrease schedule-controlled responding for food and produce thermal antinociception. (A) Baclofen alone and in combination with equi-effective doses of morphine decreases schedule-controlled behavior. (B) Morphine alone and in combination with equi-effective doses of baclofen decreases schedule-controlled behavior. (C) Baclofen alone and in combination with morphine increases latency to respond to thermal stimulus. (D) Morphine alone and in combination with baclofen increases latency to respond to thermal stimulus. Ordinate in (A and C) depicts the rate of responding calculated as a percentage of control and in (B and D) the maximum percent effect (MPE) as a percentage of latency to respond to thermal stimulus. Abscissa depicts the dose of drug administered. ${ }^{*} P<0.05 ; * * P<0.01 ; * * * P<0.001 ; * * * * P<0.0001$ vs. vehicle. Data reflect mean \pm S.E.M., $n=8 \mathrm{rats} / \mathrm{group}$.

Effects of Morphine and Buspirone on Schedule-Controlled Responding and Latency to Respond to Thermal Stimuli. Figure 2 shows the effects of morphine and buspirone on rate of FR10 responding for food and latency to respond in the hot plate test. Buspirone alone dose dependently decreased the rate of food-maintained responding (Fig. $2 \mathrm{~A} ; F_{7,28}=25.8, P<0.0001$ ). The $\mathrm{ED}_{50}$ value of buspirone to reduce operant responding was 0.57 (95\% CL: 0.31-1.07) $\mathrm{mg} / \mathrm{kg}$. We next assessed the doseresponse relationship of equi-effective doses of buspirone in combination with morphine and found a leftward shift in the dose-response relationship compared with either compound given alone (Fig. 2, A and B). Analysis of the mixture potency and additive potency revealed additivity between these drugs. The calculated experimental Zmix [7.22 $(5.23-9.21) \mathrm{mg} / \mathrm{kg}$ ] was not significantly different from the calculated theoretical Zadd [5.70 (5.68-5.72) mg/kg].

Figure 2 also shows the effects of morphine and buspirone, administered separately and together, on thermal antinociception. Buspirone dose dependently increased the latency to respond to thermal stimulus (Fig. $2 \mathrm{C} ; F_{7,28}=$ $24.0 P<0.0001$ ). The $\mathrm{ED}_{50}$ value of buspirone to produce thermal antinociception was 2.20 [95\% CL: 1.47-3.29) $\mathrm{mg} / \mathrm{kg}]$. Operant responding was 3.86 -fold more sensitive to the effects of buspirone than was thermal nociception [potency ratio: 3.86 (95\% CL: 3.07-4.65)]. We next assessed the dose-response relationship of equi-effective doses of morphine and buspirone in combination from Fig. 2, A and $\mathrm{B}$, in the hot plate test and found a leftward shift in the doseresponse relationship compared with either compound given alone (Fig. 2, C and D). Analysis of the mixture potency and additive potency revealed a synergistic interaction between these drugs. The calculated experimental Zmix [5.87 (4.35-7.39) $\mathrm{mg} / \mathrm{kg}$ ] was significantly less than the calculated theoretical Zadd [14.72 (14.66-14.78) $\mathrm{mg} / \mathrm{kg})]$ and below the line of additivity. The degree of effects from the combination of morphine and buspirone was 5.82 -fold higher in the hot plate test.

Effects of Baclofen and Buspirone on Schedule-Controlled Responding and Latency to Respond to Thermal Stimuli. Figure 3 shows the effects of baclofen and buspirone on rate of FR10 responding for food and latency to respond in the hot plate test. We assessed the doseresponse relationship of equi-effective doses of baclofen in combination with buspirone and found a leftward shift in the dose-response relationship compared with buspirone given alone (Fig. 3, A and B). Analysis of the mixture potency and additive potency revealed additivity. The calculated experimental Zmix [2.82 (2.06-3.58) $\mathrm{mg} / \mathrm{kg}]$ was not significantly different from the calculated theoretical Zadd [4.36 (3.56-5.16) mg/kg].

Figure 3 also shows the effects of buspirone and baclofen on thermal antinociception. We assessed the dose-response relationship of equi-effective doses of baclofen and buspirone in combination from Fig. $3, \mathrm{~A}$ and $\mathrm{B}$, in the hot plate test and found a leftward shift in the dose-response relationship compared with buspirone given alone (Fig. 3, C and D). Analysis of the mixture potency and additive potency revealed a synergistic interaction between these drugs. The calculated experimental Zmix [3.70 (3.00-4.40) mg/kg)] was significantly less than the calculated theoretical Zadd [7.38 $(6.14-8.62) \mathrm{mg} / \mathrm{kg}]$. The degree of effects from the 

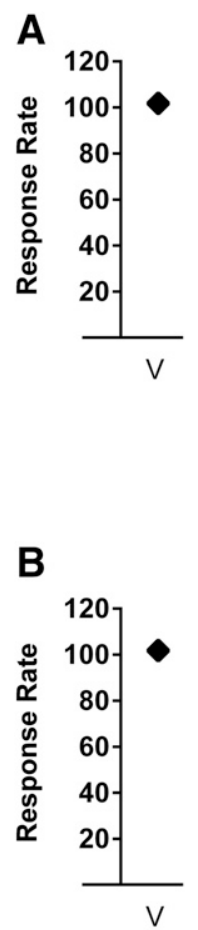
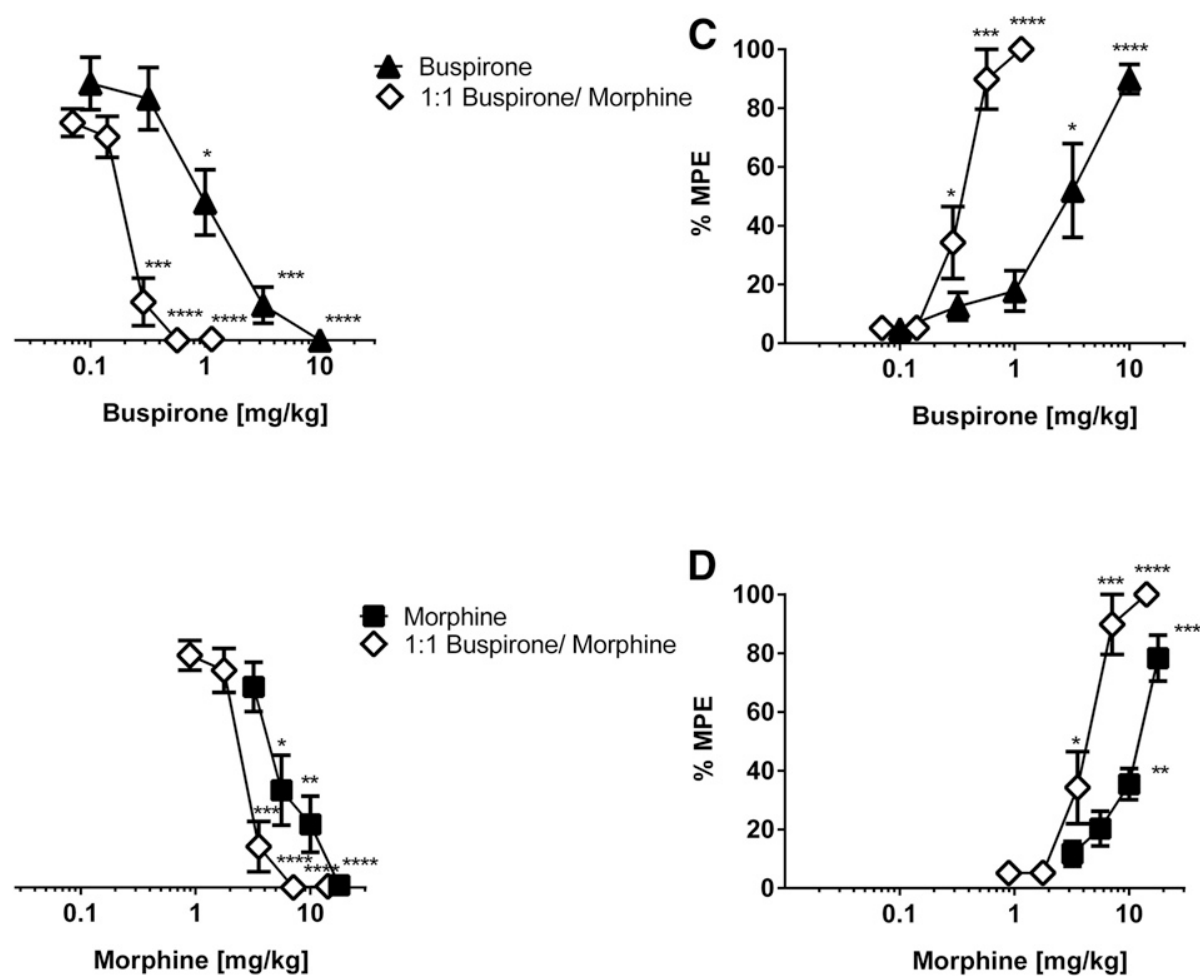

Fig. 2. Systemic buspirone and morphine decrease schedule-controlled responding for food and produce thermal antinociception. (A) Buspirone alone and in combination with equi-effective doses of morphine decreases schedule-controlled behavior in a dose-related manner. (B) Morphine alone and in combination with equi-effective doses of buspirone decreases schedule-controlled behavior. (C) Buspirone alone and in combination with morphine increases latency to respond to thermal stimulus. (D) Morphine alone and in combination with buspirone increases latency to respond to thermal stimulus. Ordinate in (A and $\mathrm{C}$ ) depicts the rate of responding calculated as a percentage of control and in (B and D) the maximum percent effect (MPE) as a percentage of latency to respond to thermal stimulus. Abscissa depicts the dose of drug administered. $* P<0.05 ; * * P<0.01 ; * * * P<0.001$; **** $P<0.0001$ vs. vehicle. Morphine dose-response curves are the same as those shown in Fig. 1 . Data reflect mean \pm S.E.M., $n=8$ rats/group.

combination of baclofen and buspirone was 2.39-fold higher in the hot plate test.

Effect of $0.032 \mathrm{mg} / \mathrm{kg}$ Naltrexone on Morphine and Morphine Drug Combination-Induced Changes in Operant Responding and Latency to Respond to Thermal Stimuli. By itself, $0.032 \mathrm{mg} / \mathrm{kg}$ naltrexone administered during the first cycle, followed by vehicle in subsequent cycles, did not alter rates of responding for food (Supplemental Fig. $1 \mathrm{C} ; F_{7,37}=3.1 ; P=0.07$ ) or latency to respond to thermal stimulus (Supplemental Fig. $1 \mathrm{D} ; F_{7,35}=1.8 ; P=0.19$ ). As indicated by a rightward shift in dose-response functions (Fig. 4, A and B), $0.032 \mathrm{mg} / \mathrm{kg}$ naltrexone antagonized the effects of morphine on operant responding and latency to respond to thermal stimulus. In the presence of naltrexone, the $\mathrm{ED}_{50}$ values of morphine to reduce operant responding and to produce thermal antinociception were 29.51 (19.23-39.79) and $50.34(41.93-58.78) \mathrm{mg} / \mathrm{kg}$, respectively. A potency ratio analysis confirmed significant antagonism of morphine by $0.032 \mathrm{mg} / \mathrm{kg}$ naltrexone, as evidenced by a 4.16 (95\% confidence limits: 3.76-5.34) potency ratio between respective morphine dose-response functions to decrease operant responding and a 3.12 (95\% CL: $2.86-3.30)$ potency ratio between respective morphine dose-response functions to produce thermal antinociception. Naltrexone antagonized the combined effects of baclofen and morphine on operant responding (Fig. 4C), as evidenced in a potency ratio of 2.78 (95\% CL: 2.35-3.28) between respective dose-response functions, as well as to produce thermal antinociception (Fig. 4D), as evidenced in a potency ratio of 1.69 (95\% CL:
1.59-1.80) between respective dose-response functions. Naltrexone also antagonized the combined effects of buspirone and morphine on operant responding (Fig. 4C) and to produce thermal antinociception with a potency ratio analysis confirming an antagonistic effect in both [1.19 (95\% CL: 1.02-1.38] and 3.52 [95\% CL: 3.08-4.00), respectively].

Effect of $320 \mathrm{mg} / \mathrm{kg}$ CGP35348 on Baclofen and Baclofen Drug Combination-Induced Changes in Operant Responding and Latency to Respond to Thermal Stimuli. By itself, $320 \mathrm{mg} / \mathrm{kg}$ CGP35348 administered during the first cycle, followed by vehicle in subsequent cycles did not alter rates of responding for food (Supplemental Fig. 1E; $\left.F_{6,30}=2.8 ; P=0.06\right)$ or latency to respond to thermal stimulus (Supplemental Fig. $1 \mathrm{~F} ; F_{6,30}=3.3 ; P=0.08$ ). As indicated by a rightward shift in dose-response functions (Fig. 5, A and B), $320 \mathrm{mg} / \mathrm{kg}$ CGP35348 antagonized the effects of baclofen on operant responding. In the presence of CGP35348, the $\mathrm{ED}_{50}$ values of baclofen to reduce operant responding and to produce thermal antinociception were $13.62(6.07-30.56)$ and $17.11(13.89-21.08) \mathrm{mg} / \mathrm{kg}$, respectively. A potency ratio analysis confirmed significant antagonism of baclofen by $320 \mathrm{mg} / \mathrm{kg}$ CGP35348, as evidenced in a potency ratio of 3.55 (95\% CL: 2.46-5.14) between respective baclofen doseresponse functions to decrease operant responding and a potency ratio of 1.96 (95\% CL: 1.66-2.30) between respective baclofen dose-response functions to produce thermal antinociception. CGP35348 significantly antagonized the combined effects of baclofen and morphine on operant responding 

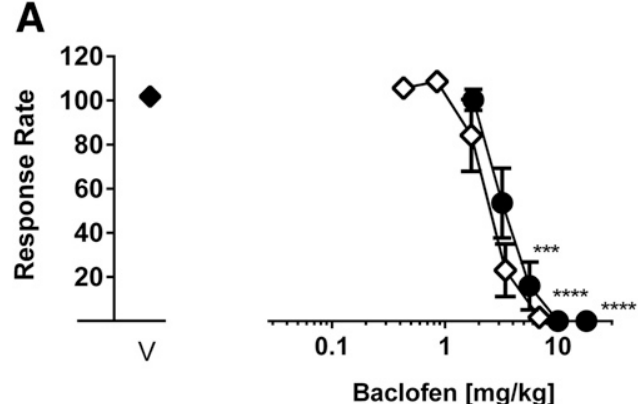

B
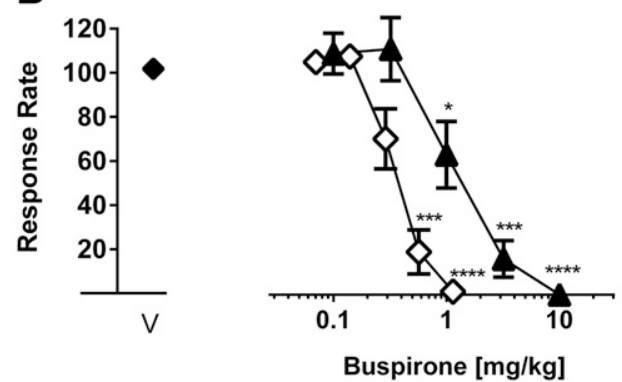
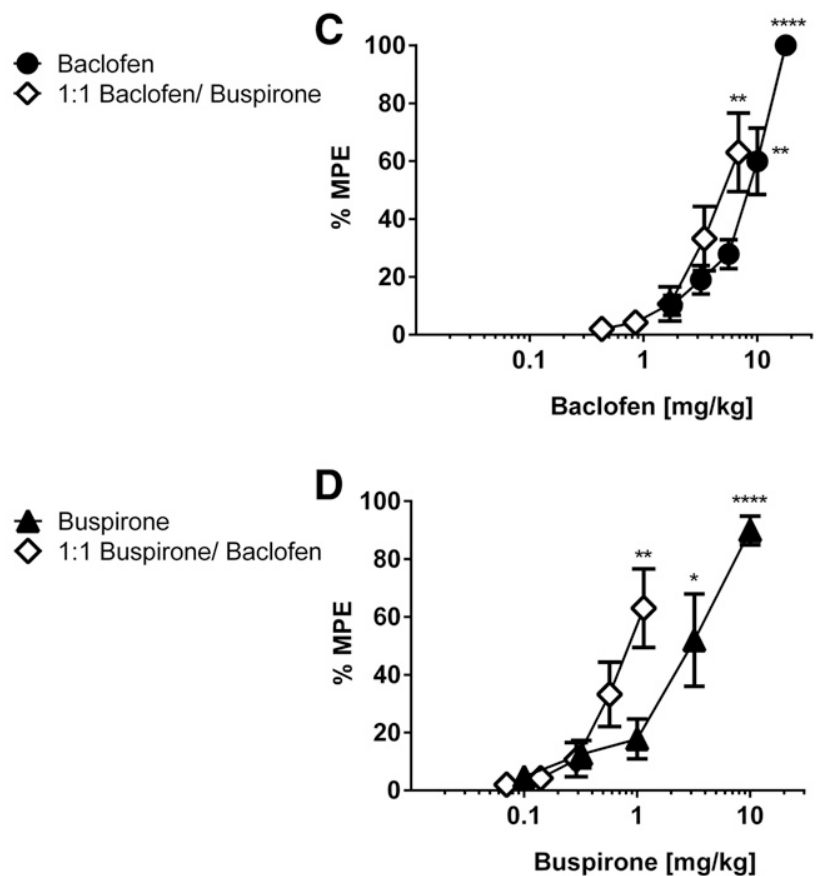

Fig. 3. Systemic baclofen and buspirone decrease schedule-controlled responding for food and produce thermal antinociception. (A) Baclofen alone and in combination with equi-effective doses of buspirone decrease schedule-controlled behavior. (B) Buspirone alone and in combination with equi-effective doses of baclofen decrease schedule-controlled behavior. (C) Baclofen alone and in combination with buspirone increased latency to respond to thermal stimulus in a dose-related manner. (D) Buspirone alone and in combination with baclofen increased latency to respond to thermal stimulus. Ordinate in (A and C) depicts the rate of responding calculated as a percentage of control and in (B and D) the maximum percent effect (MPE) as a percentage of latency to respond to thermal stimulus. Abscissa depicts the dose of drug administered. $* P<0.05 ; * * P<0.01 ; * * * P<0.001 ; * * * * P<0.0001$ vs. vehicle. Baclofen and buspirone dose-response curves are the same as those shown in Figs. 1 and 2, respectively. Data reflect mean \pm S.E.M., $n=8$ rats/group.

(Fig. 5C) and latency to respond to thermal stimulus (Fig. 5D) as evidenced in potency ratios of [1.52 (95\% CL: $1.40-1.67)$ and 1.65 (95\% CL: 1.39-1.96), respectively]. However, CGP35348 did not significantly antagonize the effects of combined baclofen and buspirone on operant responding (Fig. 5E) with a potency ratio analysis confirming no significant change in baclofen's effect [1.00 (95\% CL: 0.60-1.40]. Thermal antinociception from the highest dose combination of $6.83 \mathrm{mg} / \mathrm{kg}$ baclofen and $1.14 \mathrm{mg} / \mathrm{kg}$ buspirone was significantly antagonized by $320 \mathrm{mg} / \mathrm{kg}$ CGP35348, as indicated by the results of a $t$ test $(P<0.05)$.

Development of Tolerance to Baclofen, Morphine and Buspirone on Schedule-Controlled Responding and Thermal Nociceptive Latency. After all studies were completed, dose-response curves of baclofen, morphine, and buspirone were redetermined for each drug to decrease schedule-controlled responding and to produce thermal antinociception (Supplemental Fig. 2). Baclofen underwent significant tolerance in schedule-controlled responding (significant interaction between treatment and time: $F_{4,48}$ $=2.64 ; P<0.05)$ but did not undergo significant tolerance in the hot plate test (interaction between treatment and time: $F_{4,48}=2.64 ; P=0.78$ ) (Supplemental Fig. $2, \mathrm{~A}$ and B). Conversely, morphine did not undergo significant tolerance in schedule-controlled responding (interaction between treatment and time: $F_{3,36}=2.02 ; P=0.13$ ), but did undergo significant tolerance in the hot plate test (significant interaction between treatment and time: $F_{3,36}=3.80$; $P<0.05$ ) (Supplemental Fig. 2, C and D). Meanwhile, buspirone underwent significant sensitization in schedulecontrolled responding (significant interaction between treatment and time: $F_{4,48}=7.65 ; P<0.0001$ ), but underwent significant tolerance in the hot plate test (significant interaction between treatment and time: $F_{4,48}=3.55$; $P<0.05$ ) (Supplemental Fig. 2, E and F).

\section{Discussion}

Here we show the potency for morphine, baclofen, and buspirone to decrease schedule-controlled responding for food is higher than that needed to produce thermal antinociception. The effects of morphine plus baclofen to decrease schedulecontrolled responding for food and to produce thermal antinociception were synergistic. The effects of morphine plus buspirone and baclofen plus buspirone on schedule-controlled responding for food were additive, and the effects of the two combinations to increase the latency to respond to thermal stimulus were synergistic. As dose combination experiments were completed in a randomized order, it is difficult to determine the overall contribution to the development of tolerance or sensitivity, specifically regarding the rate-decreasing effects of buspirone, of drug effects on the observed experimental outcomes. These findings suggest that buspirone may produce synergistic acute antinociception when combined with morphine, with only additive or less-enhanced effects on other behaviors. Furthermore, these findings over the combination of baclofen plus buspirone may represent novel $\mathrm{GABA}_{\mathrm{B}}$ and $5-\mathrm{HT}_{1 \mathrm{~A}}$ receptor interactions, which might be further optimized to yield novel therapeutics for the treatment of pain with a lessened side effect profile.

A dose of naltrexone that antagonized morphine's effects in schedule-controlled responding and thermal antinociception 

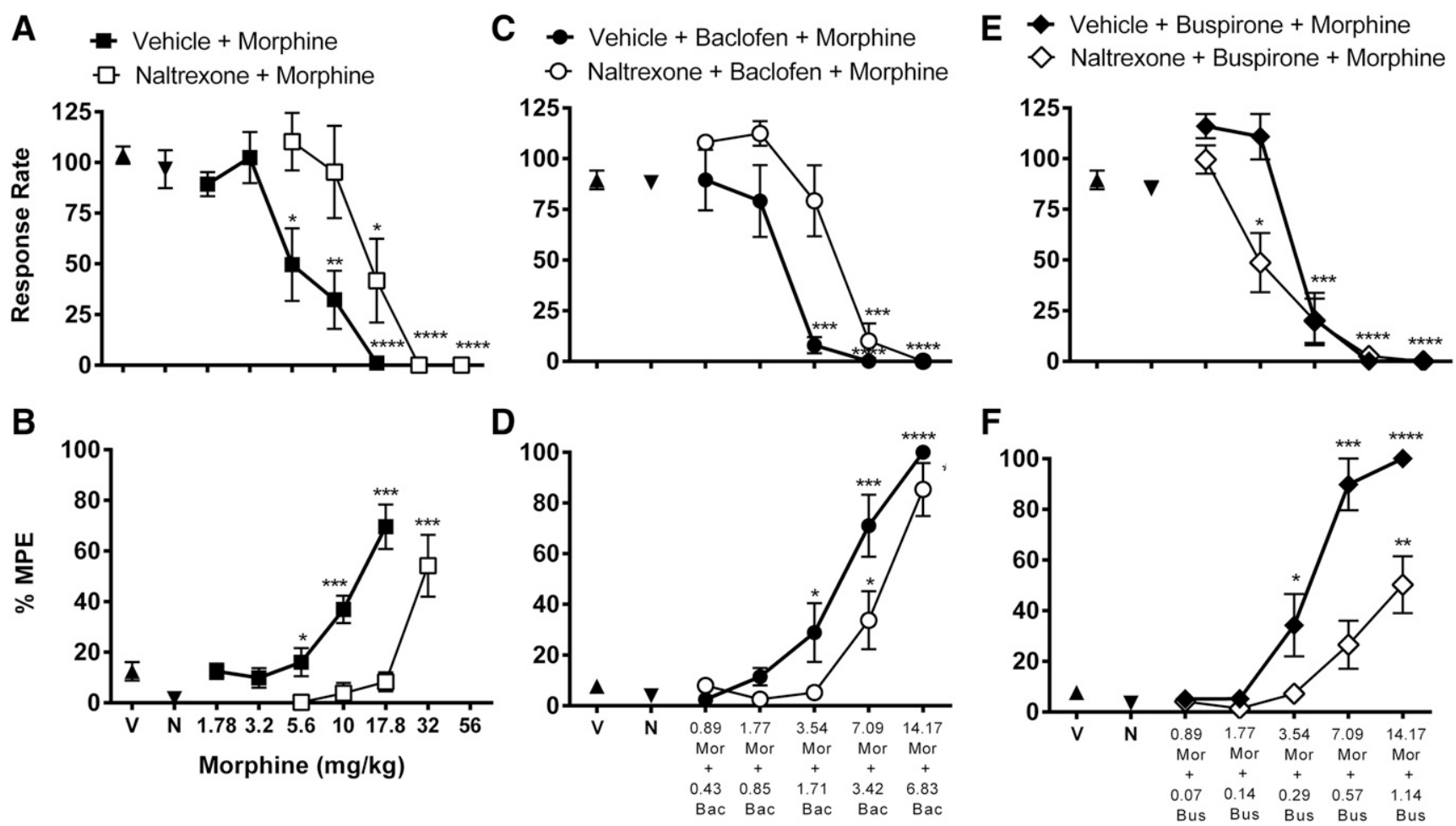

Fig. 4. Effects of pretreatment with naltrexone on drug-induced changes in food-maintained responding and thermal antinociception. Abscissae: drug dose (mg/kg, i.p.), log scale; ordinates: (A, C, and E) Percent control rates of food-maintained responding. (B, D, and F) Percent maximum possible effect in the hot plate test. (A and B) Dose-dependent effects of morphine; ( $\mathrm{C}$ and $\mathrm{D})$ equi-effective dose combinations of baclofen and morphine; and (E and F) equi-effective dose combinations of buspirone and morphine with pretreatment with naltrexone. $* P<0.05 ; * * P<0.01 ; * * * P<0.001 ; * * * * P<0.0001$ vs. vehicle or naltrexone, respectively, according to pretreatment. Morphine dose-response curves are the same as those shown in Fig. 1 . Data reflect mean \pm S.E.M., $n=8$ rats/group.

also antagonized both these behavioral effects when morphine was combined with baclofen and, separately, when morphine was combined with buspirone. Thus, opioid receptors were involved in the effects produced when morphine was combined with either baclofen or buspirone to decrease in schedule-controlled responding, as well as to produce thermal antinociception. A dose of CGP34358 that antagonized baclofen's effects in schedule-controlled responding and thermal antinociception also antagonized both these behavioral effects when baclofen was combined with morphine. However, when compared with the effects of CGP34358, naltrexone produced a greater rightward shift of the baclofen plus morphine dose-response curve in both behavioral measures. Thus, it is likely that opioid receptors played a bigger role in the effects of the baclofen plus morphine drug combination than $\mathrm{GABA}_{\mathrm{B}}$ receptors. Interestingly, this same dose of CGP34358 failed to antagonize the effects of baclofen plus buspirone on schedule-controlled responding and only antagonized the highest studied dose combination of baclofen and buspirone in the hot plate test. From this, it is likely that the actions of buspirone at multiple receptors [i.e., buspirone can act as a serotonin $(5-\mathrm{HT})_{1 \mathrm{~A}}$ receptor agonist, or a dopamine $\mathrm{D}_{2}, \mathrm{D}_{3}$, and $\mathrm{D}_{4}$ antagonist) accounts for the observed pharmacological effects of the combination of baclofen plus buspirone.

To the authors' knowledge, the examination of the combination of baclofen and buspirone on the effects of thermal antinociception or scheduled-controlled response behavior has not been reported in the literature. Here we show that the combination of baclofen and buspirone synergistically increased latency to respond to thermal stimulus and only additively disrupted schedule-controlled responding.

There are several lines of evidence that support the therapeutic combination of baclofen with morphine. There are numerous studies that suggest that $\mathrm{GABA}_{\mathrm{B}}$ agonists possess relatively low abuse potential and may in fact be useful as therapeutics to treat drug abuse. Previous studies have shown that baclofen decreases the reinforcing effects of opioids in rat self-administration procedures (Xi and Stein, 1999; Ramshini et al., 2013). Treatment with a GABAB inhibitor phaclofen seemingly increased morphine self-administration (Ramshini et al., 2013). Similarly, baclofen has been found to abolish morphine preference in the place conditioning assay in mice (Meng et al., 2014). These anti-rewarding effects of baclofen are attributed to the actions of GABAB receptor stimulation to inhibit dopamine release. Specifically, direct administration of baclofen into the ventral tegmental area has been found to inhibit the local release of opioid-induced dopamine (Klitenick et al., 1992; Xi and Stein, 1999). Systemic administration of baclofen has been found to inhibit the release of opioid-induced dopamine in the nucleus accumbens (Fadda et al., 2003; Fu et al., 2012). This baclofeninduced decrease of dopamine release in the nucleus accumbens has also been found in correlation with repeated administration of baclofen with morphine, where baclofen and morphine coadministration prevented the development of tolerance to the effects of morphine on locomotor activity in rats (Fu et al., 2012; Topkara et al., 2017). In addition to 
A

Vehicle + Baclofen

- CGP35348 + Baclofen
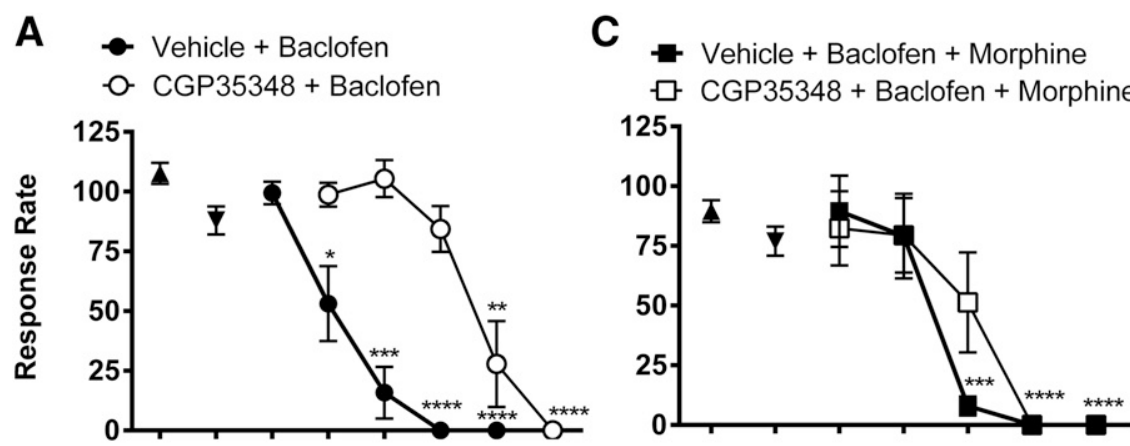

B
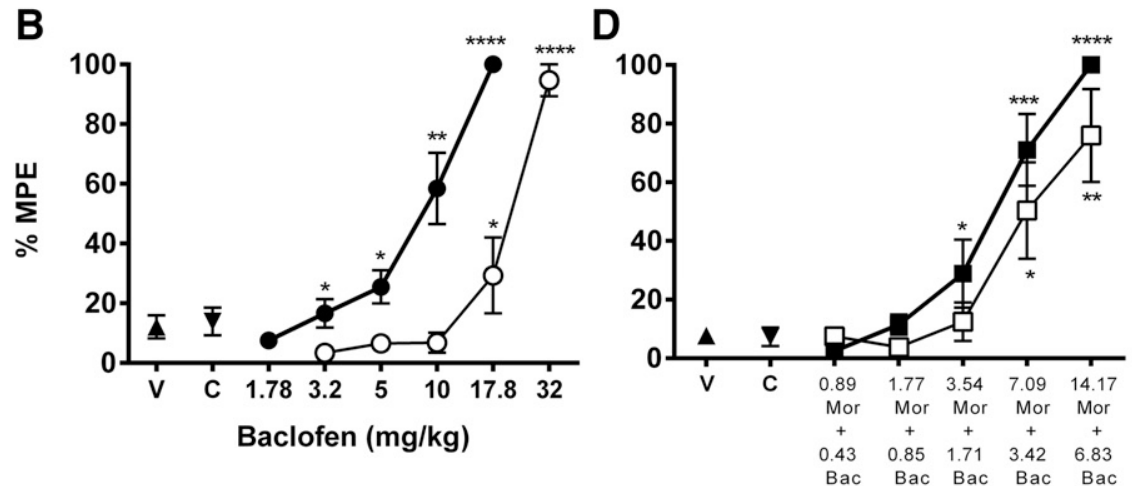

E Vehicle + Baclofen + Buspirone $\diamond$ CGP35348 + Baclofen + Buspirone

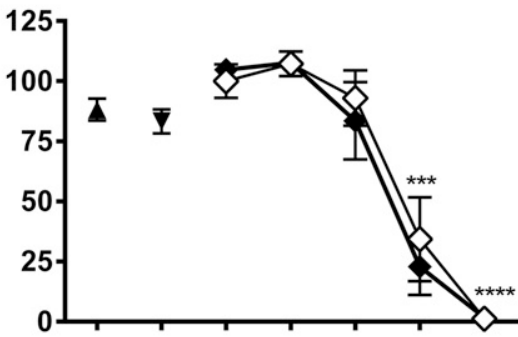

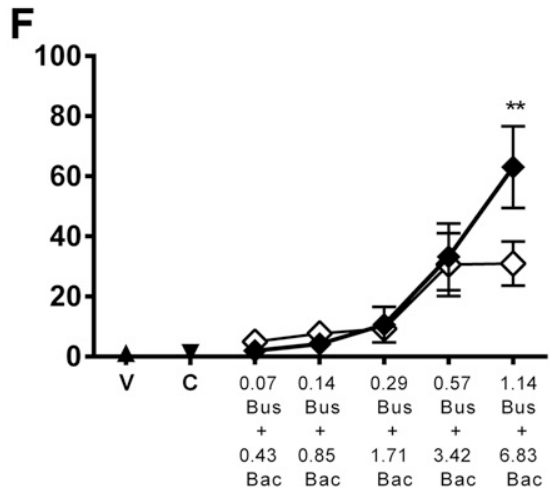

Fig. 5. Effects of pretreatment with CGP35348 on drug-induced changes in food-maintained responding and thermal antinociception. Abscissae: drug dose (mg/kg, i.p.), log scale; ordinates: (A, C, and E) percent control rates of food-maintained responding. (B, D, and F) Percent maximum possible effect in the hot plate test. (A and B) Dose-dependent effects of buspirone; (C and D) equi-effective dose combinations of baclofen and morphine; and (E and F) equi-effective dose combinations of buspirone and baclofen with $320 \mathrm{mg} / \mathrm{kg}$ CGP35348 pretreatment. $* P<0.05$; $* * P<0.01$; $* * * P<0.001$; $* * * * P<$ 0.0001 vs. vehicle or CGP35348, respectively according to pretreatment. The baclofen dose-response curves are the same as those shown in Fig. 1 . Data reflect mean \pm S.E.M., $n=7$ to 8 rats/group (one rat was lost to attrition).

directly modifying the rewarding properties of opioids, baclofen has also been found to reduce behavioral signs of naloxone-induced morphine withdrawal in mice (Pedron et al., 2016) and rats (Topkara et al., 2017). Additional studies have shown that baclofen can decrease the rewarding effects of other commonly abused drugs such as nicotine, cocaine, and methamphetamine (Ranaldi and Poeggel 2002; Fadda et al., 2003). It was previously found that baclofen decreased morphine-induced nausea and vomiting in ferrets (Suzuki et al., 2005). In a retrospective clinical study, multiple sclerosis patients that had been on intrathecal (peri-spinal) baclofen for muscle spasticity but experienced uncontrolled pathologic pain responded well with the addition of intrathecal morphine for pain, with limited side effects reported (Sadiq and Poopatana, 2007). However, our studies suggest that baclofen may not be suitable in combination with opioids for acute pain management, due to synergistic drug-induced disruptions in schedule-controlled responding.

Although limited, the existing body of literature also suggests a favorable profile of the combination of buspirone with opioids for pain management. In rats, buspirone has been found to decrease the rewarding effects of morphine in the conditioned place preference assay, prevent the development of tolerance to morphine's locomotor effects, and enhance the effects of morphine in the hot plate assay (Haleem and Nawaz, 2017). In non-human primate studies, buspirone diminished the ability of animals to detect morphine in a drug discrimination study and enhanced the effects of morphine to produce thermal antinociception in the tail-flick test (Li et al., 2011).
Although buspirone has effects at multiple receptors, it can act as a $5-\mathrm{HT}_{1 \mathrm{~A}}$ receptor agonist. It has been well established that less subtype-selective 5-HT receptor agonists produce thermal antinociception and enhance the antinociceptive effects of opioids in rodents and non-human primates (Larson and Takemori, 1977; Banks et al., 2010). A small prospective German clinical study of 12 females, 20-30 years of age, found that a single dose of oral buspirone did not alter the thermal nociceptive responses of healthy volunteer subjects compared with a single intravenous morphine dose in the same testing paradigm (Pavlakovic et al., 2009). Although the results of this study reveal a negative finding, there are a considerable number of experimental factors (i.e., relatively small homogenous experimental cohort, differing routes of drug administration, single drug doses) that limit the interpretation of the results. Furthermore, as the experimental cohort in this study was comprised solely of young, premenopausal women, estrus stage was not reported. Of importance, estrus cycle stage has been reported to influence acute pain perception in healthy women (Hellstrom and Lundberg, 2000). Thus, additional clinical studies comprised of both men and women are needed to identify if buspirone alone can produce antinociception in humans and to examine as reported in the current and other preclinical studies, whether buspirone in combination with opioids produce either additive or synergistic effects in the production of antinociception.

One caveat to our current study is that we only examined 1:1 combinations of drugs, based upon $\mathrm{ED}_{50}$ values. Differential 
dose ratio combinations (i.e., 1:3, 3:1 ratios) are valuable pharmacological assessments for drug combination optimization. Specifically, a bigger dose of a drug, based upon dose equivalence, allows for the ability to select preferable pharmacological interactions, which is useful when maximizing relative therapeutic versus side effect profiles (Foucquier and Guedj, 2015). Here we only assessed decreases in schedulecontrolled responding as a potential untoward side effect of drugs alone and in combination. Further studies, outside of the scope of the current study, are needed to examine other potential side effects of these drug combinations, specifically sedation and respiratory depression, in comparison with therapeutic pain outcomes. Another important consideration of these data is that here we examine the effects of baclofen, buspirone, morphine, and respective combinations of these drugs in a model of acute nociception. The hot plate test has relatively good predictive validity for antinociceptive compounds, especially opioids (Taber, 1973; Morgan and Christie, 2011). Importantly, rats do not exhibit grimace behaviors during acute painful stimuli such as the hot plate test (Langford et al., 2010). Thus, measuring nonreflexive acute nociceptive behaviors would require significant pain stimulation. However, cellular processes underlying behaviors associated with pathologic pain are different than those of acute nociception (De Leo et al., 2006; Milligan and Watkins, 2009; Wilkerson and Milligan, 2011) and thus results shown here for acute reflexive nociceptive inhibition may not generalize to behavioral effects observed in preclinical models of pathologic pain. Further studies are needed to examine whether these drugs in multiple combinations (i.e., 1:1, 1:3. 3:1) produce reversal of pain-evoked and pain-depressed behaviors (Negus et al., 2010; Wilkerson et al., 2018) in preclinical models of pathologic pain.

Here we show that effects of the combination of morphine plus baclofen to decrease schedule-controlled responding for food and to produce thermal antinociception were synergistic, with a 3.87-fold higher degree of effect for an increased effect in thermal latency than a decreased effect in schedule-controlled responding. Meanwhile, effects of the combination of morphine plus buspirone and baclofen plus buspirone on schedulecontrolled responding for food were additive, and the effects of the two combinations to increase latency to respond to thermal stimulus were synergistic, with a 5.82- and 2.39-fold higher degree of effect in the hot plate assay, respectively. Synergistic antinociceptive effects, in conjunction with additive behaviorally disruptive effects, highlight the utility of opioid and non-opioid drug combinations as effective pain therapeutics.

\section{Acknowledgments}

The authors thank Dr. Hannah Harris, Ms. Lisa Wilson, and Ms. Ariana Brice for their technical assistance.

\section{Authorship Contributions}

Participated in research design: Wilkerson, McMahon.

Conducted experiments: Felix, Restrepo.

Contributed new reagents or analytic tools: Ansari, Coop.

Performed data analysis: Wilkerson, McMahon.

Wrote or contributed to the writing of the manuscript: Wilkerson, McMahon.

\section{References}

Banks ML, Rice KC, and Negus SS (2010) Antinociceptive interactions between Muopioid receptor agonists and the serotonin uptake inhibitor clomipramine in rhesus monkeys: role of Mu agonist efficacy. J Pharmacol Exp Ther 335:497-505.
Corsello SM, Bittker JA, Liu Z, Gould J, McCarren P, Hirschman JE, Johnston SE, Vrcic A, Wong B, Khan M, et al. (2017) The Drug Repurposing Hub: a nextgeneration drug library and information resource. Nat Med 23:405-408.

Cunningham CS and McMahon LR (2011) The effects of nicotine, varenicline, and cytisine on schedule-controlled responding in mice: differences in $\alpha 4 \beta 2$ nicotinic receptor activation. Eur J Pharmacol 654:47-52.

De Leo JA, Tawfik VL, and LaCroix-Fralish ML (2006) The tetrapartite synapse: path to CNS sensitization and chronic pain. Pain 122:17-21.

Deseure K and Hans GH (2017) Differential drug effects on spontaneous and evoked pain behavior in a model of trigeminal neuropathic pain. J Pain Res 10:279-286.

Eddy NB and Leimbach D (1953) Synthetic analgesics. II. Dithienylbutenyl- and dithienylbutylamines. J Pharmacol Exp Ther 107:385-393.

Fadda P, Scherma M, Fresu A, Collu M, and Fratta W (2003) Baclofen antagonizes nicotine-, cocaine-, and morphine-induced dopamine release in the nucleus accumbens of rat. Synapse 50:1-6.

Fields HL (2011) The doctor's dilemma: opiate analgesics and chronic pain. Neuron 69:591-594.

Foucquier J and Guedj M (2015) Analysis of drug combinations: current methodological landscape. Pharmacol Res Perspect 3:e00149.

Froestl W, Mickel SJ, Von Sprecher G, Strub D, Baumann PA, Brugger F, Gentsch C, Jaekel J, Olpe HR, Rihs G, et al. (1995) Phosphinic acid analogues of GABA: 2. Selective, orally active GABA ${ }_{\mathrm{B}}$ antagonists. $J$ Med Chem 38:3313-3331.

Fu Z, Yang H, Xiao Y, Zhao G, and Huang H (2012) The $\gamma$-aminobutyric acid type B $(\mathrm{GABAB})$ receptor agonist baclofen inhibits morphine sensitization by decreasing the dopamine level in rat nucleus accumbens. Behav Brain Funct 8:20.

Haleem DJ and Nawaz S (2017) Inhibition of reinforcing, hyperalgesic, and motor effects of morphine by buspirone in rats. J Pain 18:19-28.

Hellström B and Lundberg U (2000) Pain perception to the cold pressor test during the menstrual cycle in relation to estrogen levels and a comparison with men. Integr Physiol Behav Sci 35:132-141.

Hwang JH and Yaksh TL (1997) The effect of spinal GABA receptor agonists on tactile allodynia in a surgically-induced neuropathic pain model in the rat. Pain $\mathbf{7 0}$ : $15-22$

Ignatowska-Jankowska B, Wilkerson JL, Mustafa M, Abdullah R, Niphakis M, Wiley JL, Cravatt BF, and Lichtman AH (2015) Selective monoacylglycerol lipase inhibitors: antinociceptive versus cannabimimetic effects in mice. J Pharmacol Exp Ther 353:424-432.

Kilkenny C, Browne W, Cuthill IC, Emerson M, and Altman DG; NC3Rs Reporting Guidelines Working Group (2010) Animal research: reporting in vivo experiments: the ARRIVE guidelines. Br J Pharmacol 160:1577-1579.

Klitenick MA, DeWitte P, and Kalivas PW (1992) Regulation of somatodendritic dopamine release in the ventral tegmental area by opioids and GABA: an in vivo microdialysis study. J Neurosci 12:2623-2632.

Korneyev AY and Seredenin SB (1993) Effect of 5-HT-1A receptor agonists on paw licking reaction on hot plate and unpunished drinking in rat. Life Sci 52.997-1004.

Langford DJ, Bailey AL, Chanda ML, Clarke SE, Drummond TE, Echols S, Glick S, Ingrao J, Klassen-Ross T, Lacroix-Fralish ML, et al. (2010) Coding of facial expressions of pain in the laboratory mouse. Nat Methods 7:447-449.

Larson AA and Takemori AE (1977) Effect of fluoxetine hydrochloride (Lilly 110140), a specific inhibitor of serotonin uptake, on morphine analgesia and the development of tolerance. Life Sci 21:1807-1811.

Li JX, Koek W, Rice KC, and France CP (2011) Effects of direct- and indirect-acting serotonin receptor agonists on the antinociceptive and discriminative stimulus effects of morphine in rhesus monkeys. Neuropsychopharmacology 36:940-949.

McGrath JC, Drummond GB, McLachlan EM, Kilkenny C, and Wainwright CL (2010) Guidelines for reporting experiments involving animals: the ARRIVE guidelines. Br J Pharmacol 160:1573-1576.

McMahon LR and Cunningham KA (2001) Antagonism of 5-hydroxytryptamine(2a) receptors attenuates the behavioral effects of cocaine in rats. J Pharmacol Exp Ther 297:357-363.

McMahon LR and France CP (2002) Acute and chronic effects of the neuroactive steroid pregnanolone on schedule-controlled responding in rhesus monkeys. Behav Pharmacol 13:545-555.

Meng S, Quan W, Qi X, Su Z, and Yang S (2014) Effect of baclofen on morphineinduced conditioned place preference, extinction, and stress-induced reinstatement in chronically stressed mice. Psychopharmacology (Berl) 231:27-36.

Milligan ED and Watkins LR (2009) Pathological and protective roles of glia in chronic pain. Nat Rev Neurosci 10:23-36.

Morgan MM and Christie MJ (2011) Analysis of opioid efficacy, tolerance, addiction and dependence from cell culture to human. Br J Pharmacol 164:1322-1334.

National Research Council (2011) Guide for the Care and Use of Laboratory Animals, National Academies Press, Washington, DC.

Negus SS, Bilsky EJ, Do Carmo GP, and Stevenson GW (2010) Rationale and methods for assessment of pain-depressed behavior in preclinical assays of pain and analgesia. Methods Mol Biol 617:79-91.

Pavlaković G, Tigges J, and Crozier TA (2009) Effect of buspirone on thermal sensory and pain thresholds in human volunteers. BMC Clin Pharmacol 9:12

Pedrón VT, Varani AP, and Balerio GN (2016) Baclofen prevents the elevated plus maze behavior and BDNF expression during naloxone precipitated morphine withdrawal in male and female mice. Synapse 70:187-197.

Ramshini E, Alaei H, Reisi P, Alaei S, and Shahidani S (2013) The role of GABAB receptors in morphine self-administration. Int J Prev Med 4:158-164.

Ranaldi R and Poeggel K (2002) Baclofen decreases methamphetamine selfadministration in rats. Neuroreport 13:1107-1110.

Sadiq SA and Poopatana CA (2007) Intrathecal baclofen and morphine in multiple sclerosis patients with severe pain and spasticity. J Neurol 254:1464-1465.

Salte K, Lea G, Franek M, and Vaculin S (2016) Baclofen reversed thermal place preference in rats with chronic constriction injury. Physiol Res 65:349-355.

Suzuki T, Nurrochmad A, Ozaki M, Khotib J, Nakamura A, Imai S, Shibasaki M, Yajima Y, and Narita M (2005) Effect of a selective GABA(B) receptor agonist 
baclofen on the mu-opioid receptor agonist-induced antinociceptive, emetic and rewarding effects. Neuropharmacology 49:1121-1131.

Taber RI (1973) Predictive value of analgesic assays in mice and rats. Adv Biochem Psychopharmacol 8:191-211.

Tallarida RJ (2001) Drug synergism: its detection and applications. J Pharmacol Exp Ther 298:865-872.

Tallarida RJ (2006) An overview of drug combination analysis with isobolograms. J Pharmacol Exp Ther 319:1-7.

Topkara B, Yananli HR, Sakall E, and Demirkapu MJ (2017) Effects of injection of gamma-aminobutyric acid agonists into the nucleus accumbens on naloxoneinduced morphine withdrawal. Pharmacology 100:131-138.

Wilkerson JL, Curry ZA, Kinlow PD, Mason BL, Hsu KL, van der Stelt M, Cravatt $\mathrm{BF}$, and Lichtman AH (2018) Evaluation of different drug classes on transient sciatic nerve injury-depressed marble burying in mice. Pain 159:1155-1165.

Wilkerson JL, Ghosh S, Mustafa M, Abdullah RA, Niphakis MJ, Cabrera R, Maldonado RL, Cravatt BF, and Lichtman AH (2017) The endocannabinoid hydrolysis inhibitor SA-57: intrinsic antinociceptive effects, augmented morphine-induced antinociception, and attenuated heroin seeking behavior in mice. Neuropharmacology 114:156-167.
Wilkerson JL and Milligan ED (2011) The central role of glia in pathological pain and the potential of targeting the cannabinoid 2 receptor for pain relief. ISRN Anesthesiol 2011:1-23.

Wilkerson JL, Niphakis MJ, Grim TW, Mustafa MA, Abdullah RA, Poklis JL, Dewey WL, Akbarali H, Banks ML, Wise LE, et al. (2016) The selective monoacylglycerol lipase inhibitor MJN110 produces opioid-sparing effects in a mouse neuropathic pain model. J Pharmacol Exp Ther 357:145-156.

Xi ZX and Stein EA (1999) Baclofen inhibits heroin self-administration behavior and mesolimbic dopamine release. J Pharmacol Exp Ther 290:1369-1374.

Zemoura K, Ralvenius WT, Malherbe P, and Benke D (2016) The positive allosteric GABAB receptor modulator rac-BHFF enhances baclofen-mediated analgesia in neuropathic mice. Neuropharmacology 108:172-178.

Address correspondence to: Dr. Jenny L. Wilkerson, P.O. Box 100487, Department of Pharmacodynamics, College of Pharmacy, University of Florida, Gainesville, FL 32610. E-mail: jenny.wilkerson@cop.ufl.edu 